\title{
QoS-oriented Admission Control in HSDPA networks
}

\author{
Gustavo Florido \\ NQaS Research Group, Department of Electronics and Telecommunications. \\ University of the Basque Country (UPV/EHU) \\ Emails: gustavoflorido@gmail.com \\ Fidel Liberal \\ NQaS Research Group, Department of Electronics and Telecommunications. \\ University of the Basque Country (UPV/EHU) \\ Emails: fidel.liberal@ehu.es \\ José Oscar Fajardo \\ NQaS Research Group, Department of Electronics and Telecommunications. \\ University of the Basque Country (UPV/EHU) \\ Emails: joseoscar.fajardo@ehu.es
}

\begin{abstract}
HSDPA defines a new High Speed Shared Channel (HS-DSCH) which allows for practical speeds up to $10 \mathrm{Mbps}$. Among all the changes introduced by HSDPA, the most prominent ones regarding QoS are the inclusion of "link adaptation", "Hybrid ARQ" and the quick "Scheduling" algorithms. In HSDPA the Radio Network Controller (RNC) is still the responsible for admitting new users but also for handling different kinds of traffics of different users over the same channel. One of the mostly adopted solutions for admitting new users assuring QoS comprises an admission control algorithm based on performance, with the cells power as the limiting resource. This kind of algorithms makes use of a static "guard power offset" for users whose channel conditions worsen in the course of the connection. In this paper, we propose two admission control algorithms that take into account that this guard power varies according to the channel conditions of each user, the number of allowed users, the service and the transmission speed that they require, and the power used by the cell on each Transmission Time Interval (TTI).

The coverage radius of the cell will be divided into three concentric rings, and those users with better channel conditions (the ones nearer to the Node-B) will be assigned with higher priority. Since they will need less power than those farther they will have a better chance on satisfying their needs. Simulation scenario in Matlab shows the behavior of these proposals by comparing how many users are admitted and how many of them are satisfied.
\end{abstract}

Keywords: HSDPA, CAC, scheduling, cell coverage 


\section{Introduction}

The mobile networks have had a large growth in the past few years which makes them of great interest for performance related studies. Everyday there are more and more innovative services that require networks that offer mobility and high speed transmissions. Nowadays, HSDPA is the standard that covers the access to UMTS networks with effective speeds up to 14 Mbps. The standard HSDPA made up part of the 3GPP's Release 5 [1] in order to improve radio resources management in Release 99 (UMTS). HSDPA introduces several changes in the UTRAN including the decrease of the TTI's length to $2 \mathrm{~ms}$, adaptative modulation and codification to the immediate radio channel conditions (AMC), the H-ARQ retransmission scheme and the quick scheduling methods that also adapts to the current radio channel conditions [2]. All of these enhancements in the standard change indeed the basic principles of the access network itself and therefore, the approach followed by legacy UMTS admission control algorithms should be revisited and probably optimized in order to cope with the new environment.

There is a large significance on the study of these networks, since they are actual already developed networks. Above all the research community has largely focused on the optimization possibilities of these networks, especially on the radio resources management level, that can be translated on software changes (therefore very low cost and easy to implement at high scale).

For example in [3], authors proposed an admission control algorithm based on the user's QoS. This proposal was aimed at guaranteeing the QoS based on the power that each user requires constrained by the total power required for all of the allowed users which could not exceed the available power for the HSDPA cell. The algorithm suggested using a fixed guar power that will be used by those users that suffer some degradation on their channel conditions and therefore require more power than the initially estimated. In [4], authors proposed assigning higher priority in the admission control algorithms to those users nearest to the node-B (and therefore with better channel conditions).

To the best of our knowledge, none of the existing works considers the possibility of having a time variable guard power, which can adapt to the number of allowed users, the channel conditions and the channel resources required by the users at each TTI. In this paper, we propose two ways of varying this guard power so that it adapts better to the channel's conditions and the number of allowed users can increase without affecting those already active.

In this study we combine the idea in [4] and the algorithms proposed in [3] in order to propose an adaptative guard power based Call Admission Control (CAC) in HSDPA which will increase the number of admitted calls while maintaining the satisfactions ratio (i.e. number of admitted users that do not experience actual QoS degradation).

The rest of the paper is organized as follows: In Section 2 a brief overview of RRM algorithms in HSDPA will be provided. In Section 3 we will describe different proposals of call admission algorithm based on time varying power offset dimensioning. In Section 4 a 
distance based priority allocation scheme will be explained. Finally Section 5 will summarize simulation scenario and results and Section 6 will collect main conclusions of our research work.

\section{Radio Resource Management in HSDPA}

In wireless networks resource management algorithms define the way the system assigns the scarce radio resources. On HSDPA, this management is made in the UTRAN, through algorithms located on the RNC as well as on the Node-B. These algorithms try to assure the coverage area planned for each service along with the link quality required, with the maximum capacity of the cell by allowing a small amount of canceled new calls, low handover and low dropped calls These standardized Radio Resource Management algorithms (RRM) in HSDPA are depicted in Figure 1.

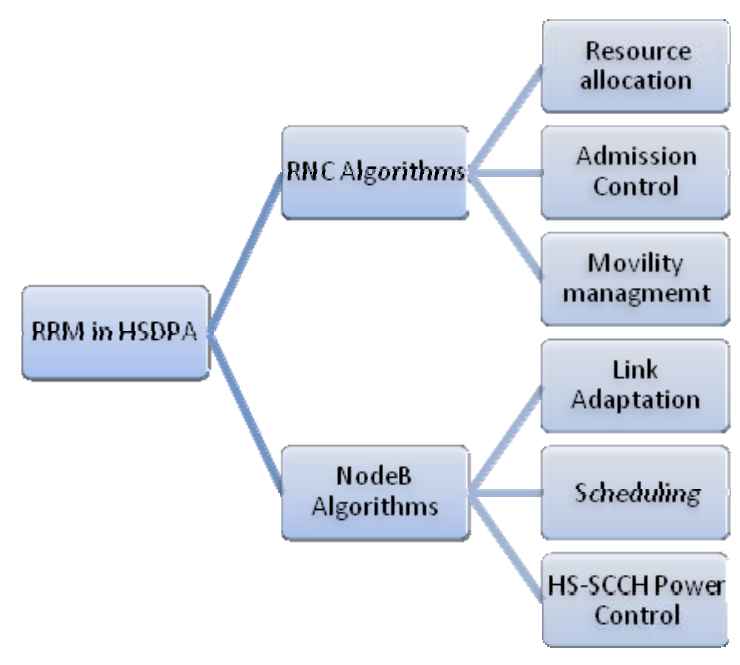

Figure 1. RRM in HSDPA.

In any access network the call admission control decides whether or not to admit a new user given the condition that they can establish their services without worsening the conditions of already active users. For different kinds of services, different resources can be critical or not. For example, for a VoIP call established in the HS-DSCH shared channel, the most critical resource could be the e2e delay, while for other kind of service such as FTP downloads or any other non real time service, the most critical resource can be the bit rate, that, in the case of HSDPA, is directly proportional to the transmission power of the B Node.

If the demanded Guaranteed Bit Rate (GBR) of a user increases, he/she will require more frequently additional resources to be assigned to him (by the scheduler), which leads that along a time period, the user consumes more power than the available in the B Node.

Explained behavior can be better observed in the general call control admission model that uses the mechanism based on thresholds [5]. This CAC (Call Admission Control) keeps track of a series of resources being used at a specific moment $\mathrm{I}$, and estimates those at $\Delta \mathrm{I}$ by considering how many resources the call is supposed to demand, then (1):

$$
\mathrm{I}+\Delta \mathrm{I}<\mathrm{I}_{\mathrm{th}}
$$


Where $1_{\text {th }}$ is the maximum threshold of used resources and can be static or dynamic.

\subsection{Position- and user mobility- aware algorithms}

In [6] a CAC algorithm is proposed based on the UE's location with regard to the cell. Part of this principle is that a terminal far from the Node-B will experience more interference than a terminal nearer the Node- $\mathrm{B}$, and therefore, will require more resources from the cell. Then, they will be assigned pre-defined priorities depending on the class (SPI) and the location so that "nearer" calls will have higher priority.

\subsection{QoS Based Algorithms}

One of the basic control admission algorithms based on guaranteeing the QoS and based on the power as a limiting resource, is the one proposed on [3]. In this case, the algorithm considers the QoS required by the new connection, its power mapping and the constraint that it won't affect the QoS of the connections already established that have the same SPI (Scheduling Priority). Usually real time services have a higher priority since any failure in the fulfillment of their QoS requirements has a big impact into the clients. Similarly, different services will require different GBRs, which means different levels of required power, which at the same time, will vary depending on the user's position at each moment. The higher the user's GBR is, the more frequently the user will require being planned by the Scheduler. This constant re-planning results on a higher use of the cell's resources like the power. For certain GBR, the required power of a user can also vary accordingly to the radio channel's conditions, which is directly linked to the location of the user with regard of the Node-B. The better the radio channel's conditions are, the higher the CQI is, which means that the cell will transmit with a higher order of modulation having a bigger transport block, and the scheduler will be required to plan the user less frequently, so that less resources will be needed.

The CAC will work following the inequality in (2):

$$
P_{H S D A} \geq P_{n}+\sum_{x \geq S I_{n}} P_{S P I}(x)+P_{S C C H}+P_{o}
$$

Where if the power assigned for HSDPA is greater than the sum of the power used by the active users with SPI (or higher) priority, the power used by the control channel HS-SCCH, the power required by the new user and a guard power factor, the call will be admitted, otherwise, it will be blocked by the cell.

\subsection{Scheduling using Strict Channel Allocation policies}

It is important that both scheduling and admission control algorithms are integrated in their policies and approaches: CAC algorithms make sure that existing resources are enough to guarantee the QoS requirements of the new call. But meanwhile scheduling algorithms are responsible for the allocation of resources and if this is not made on the right way, the call won't have guaranteed its QoS requirements. In [3] an AC algorithm that make sure that the cell has enough power to guarantee QoS to the new call is proposed, and in [7], a scheduling algorithm that shares and allocates this power is studied. So, although it is the admission control algorithm's job to ensure that all admitted users can be satisfied, situations could arise 
due to user mobility when such guarantees cannot be made and other approaches should be considered (see another example in [8]).

Generally, two user classes are considered with different GBR and different SPI. For example, even though the problem can be generalized, in [7] two classes are proposed, Gold Class and Silver Class.

Common scheduling policies (such as those in [7]) are as follows:

1. A silver user can be scheduled only if all gold users have been satisfied.

2. If there are multiple gold users with throughput less than GBR, the gold user with the highest value of CQI is scheduled.

3. A silver user with throughput less than GBRs has higher priority than any gold or silver user that meets its GBR.

4. If there are multiple silver users with throughput less than GBRs while all gold users have been satisfied, a silver user with the highest value of CQI is scheduled.

5. When all users have met their GBR, surplus capacity must be proportionally distributed among the gold and silver users under similar conditions according to their GBRs.

6. As a result of these policies, satisfied user probability is maximized because resources are allocated for each user get what it really need.

\section{Proposal of Admission Control based on quality, with time-varying power reservation}

We propose two different approaches in order to carry out time-varying power reservation according to instantaneous conditions of users and the cell. As we mentioned before, admission control algorithm proposed in [3] made a power reservation with a fixed amount of guard power reserved for admitted users, in case that they need more power than the previously estimated by the AC. This power is called Power Offset. In [3], Pedersen considers a fixed amount of power in every moment, regardless of how many users are connected, what are their channel conditions or what their QoS requirements are.

\section{1 $1^{\text {st }}$ Proposal. Power reservation according to the average CQI perceived by the user.}

This approach consists in computing the average CQI perceived by the user during that time interval, using the same window time used by the scheduler.

A user with a certain GBR and a certain CQI will need a certain amount of cell power. If that user wants to maintain the same service level he/she could require more cell power if its channel conditions get worse, which would be caused in most cases by a user's movement. The first calculation made in this algorithm is the average CQI perceived by the user during N TTIs (3).

$$
\overline{C Q I}_{k}^{t}=\frac{1}{N} \sum_{i=1}^{N} C Q I_{k}^{i}
$$


Where, $\overline{C Q I}_{k}^{T T I}$ is the average CQI for user $\mathrm{k}$ in instants $\mathrm{t}, \mathrm{N}$ is the windows size in TTIs and $C Q I_{k}^{i}$ is the CQI perceived by user $\mathrm{k}$ in time $\mathrm{i}$.

Later the estimated required power for user $k$ to guarantee its GBR is calculated by (4).

$$
\text { Pot req } q_{k}^{v}=P_{H S D P A} \cdot \frac{G B R_{k}}{\text { Throughput }_{k}^{v}}
$$

Where Pot req $q_{k}^{t}$ is the estimated required power for user $\mathrm{k}$ in window $\mathrm{v}, \mathrm{W}$ is the Node B HSDPA allocated power $G B R_{k}$ is the guaranteed bit rate required by user $\mathrm{k}$ and Throughput ${ }_{k}^{v}$ provides a mapping from $\overline{C Q I}_{k}^{t}$ to the bit rate that the user would receive with those channel conditions.

In the same way the required power, if the user perceive worse channel quality conditions, is calculated. The worse channel quality is expressed by $\overline{C Q I}_{k}^{t}-n$, where $n$ is the number of CQI that the operator estimate that the channel quality can be degraded.

Finally, Power Offset for each TTI is (5):

$$
\boldsymbol{P}_{\boldsymbol{o}_{\boldsymbol{k}}}^{\boldsymbol{t}}=\text { Pot req }{ }_{k}^{v}-\text { Pot req } q_{k}^{v}
$$

Where, $\boldsymbol{P}_{\boldsymbol{o}_{\boldsymbol{k}}}^{\boldsymbol{t}}$ is the reserved power for user $\mathrm{k}$ in instant $\mathrm{t}$, Pot req ${ }_{k}^{\prime}$, is the required power for user $\mathrm{k}$ in case that channel conditions get degraded in n CQIs and Pot req $q_{k}^{v}$ is the estimated required power for each user with channel conditions presented on time window $\mathrm{v}$.

The power offset used by the admission control algorithm would be the sum of the reservation power of all active users with the same or higher priority (see (6)).

$$
\boldsymbol{P}_{\boldsymbol{o}} \text { Total }=\sum_{x \geq S P I} \boldsymbol{P}_{\boldsymbol{o}_{\boldsymbol{k}}}^{\boldsymbol{t}}
$$

The result algorithm expression is shown in (7):

$$
P_{H S D P A}=P_{\text {new }}+\sum_{x \geq S P I} P_{S P I}(x)+P_{S C C H}+\boldsymbol{P}_{\boldsymbol{o}} \text { Total }
$$

\section{$3.22^{\text {nd }}$ Proposal. Power reservation according to Node-B used Power}

For this proposal, power offset is also calculated for each user in every TTI. The proposition consists in making a power reservation equal to a percentage of the power used for each user in the last window of N TTIs. For this calculation we used the equation in (8).

$$
\operatorname{Pused}_{k}^{v}=\frac{1}{N} \sum_{m=1}^{M_{k}} P_{k}(m)
$$

Where Pused $_{k}$ is the used power for user $k$ during window $v, \mathrm{~N}$ is the size of that window and $\mathrm{m}$ is the number of TTI where the user $k$ was scheduled. $P_{k}(m)$, is the power allocated to user $k$ in TTI $\mathrm{m}, M_{k}$ is the number of times that user $k$ was scheduled on N TTIs. 
Then, Power Offset $\boldsymbol{P}_{\boldsymbol{o}}$ is calculated by (9):

$$
\boldsymbol{P}_{\boldsymbol{o}}=\operatorname{Pused}_{k}^{v} \cdot F
$$

Where, $\mathrm{F}$ is a factor that would be configured by the operator depending on its QoS policies.

\section{A Proposal for an Admission Control Algorithm based on user's position}

The next proposal is based in [6], where the user nearer to the cell has a higher priority than the user that is farther. This proposal aims at providing a method to implement this principle into a QoS-based AC algorithm. This is made by modifying the AC algorithm expression where the used power is calculated. The modification consists in calculating just the power used by user with the same or higher priority but located nearer the Node-B. We propose to divide our coverage cell ratio into three rings and just take into account user located in the same ring or in a closer ring to the Node-B.

This algorithm clearly gives more advantage to users located closer to the Node-B, which would have higher probability to being satisfied than users more remote.

On the other hand, these users would require less power than user located farther what means that we are increasing cell capacity, reducing our cell coverage ratio, in cases where a high amount of users are using the HSDPA services on the Node B. In those cases where our cell won't be saturated, users far from the cell won't be affected by this algorithm. Power Offset is calculated then as it is shown on the expression (10).

$$
\boldsymbol{P}_{\boldsymbol{o}} \text { Total }=\sum_{x \leq \text { ring }}^{x \geq \operatorname{SPI}} \boldsymbol{P}_{\boldsymbol{o}}^{\boldsymbol{t}}
$$

\section{Simulation}

A Matlab program has been developed that simulates HS-DSCH channel behavior, packet scheduling and admission control functions. We considered the following issues to have the most realistic results possible.

1. Call scenario: we have 30 HSDPA users divided in two classes. 15 users are for class 1 (higher priority) and 15 users are for class 2.

2. Users' class: we have two classes. Class 1 with higher priority, simulate streaming services users. They have a Guaranteed Bit Rate (GBR) of 256 Kbps. Class 2 represent lower priority users with a GBR og $128 \mathrm{Kbps}$ for FTP downloading service or web services. This scenario is similar to the one in [6].

3. UEs categories. We choose users of categories 7 and 8 as in [6].

4. Calls Initiations: calls are generated according to a random uniform process during the first 20 seconds and the length of each simulation is 40 seconds. 
5. Mobility pattern: we used mobility patterns provided by the Eurane project that are the same files used by NS-2 software for HSDPA simulations, and represent a pedestrian movement.

6. Spatial users' distribution: we use uniform spatial distribution of users around the coverage ratio of the cell.

7. Scheduling: we considered the scheduling policies explained earlier.

8. Cell Power: we considered a fixed HSDPA power of 10 Watts, as they did in (3).

9. Code multiplexing: for simplicity purposes we do not use users code multiplexing. Our scheduler allocates all HSDPA power to just one user every TTI.

We ran several simulations in order to study the performance of the three call admission control proposals described in this paper. First of all, we studied the algorithms' behavior without assigning higher priority to the users closest to the Node-B, and later, we gave higher priority to those users, and studied the algorithms' behavior with that kind of priority.

In Figure 2, we show the algorithms behavior, with and without priority based in user's location. Note that by considering users' location we increase both the number of admitted users and satisfied users. Also note that the number of unsatisfied users is also larger but in a lesser extent than satisfied users, especially when higher input parameters are considered.

Similarly, Figure 3 compares the number of satisfied users for different values of admitted users in each of the three algorithms (for different input parameter). Here we observe that the algorithm that carries out time-varying power reservations using the average levels of channel quality indicator improves the traditional one. This algorithm presents the best performance when it is combined with position based priority, reaching up to 9.5 satisfied calls (SPI=1) form 12 admitted calls. These results were obtained using 2 guards CQI as an input parameter.

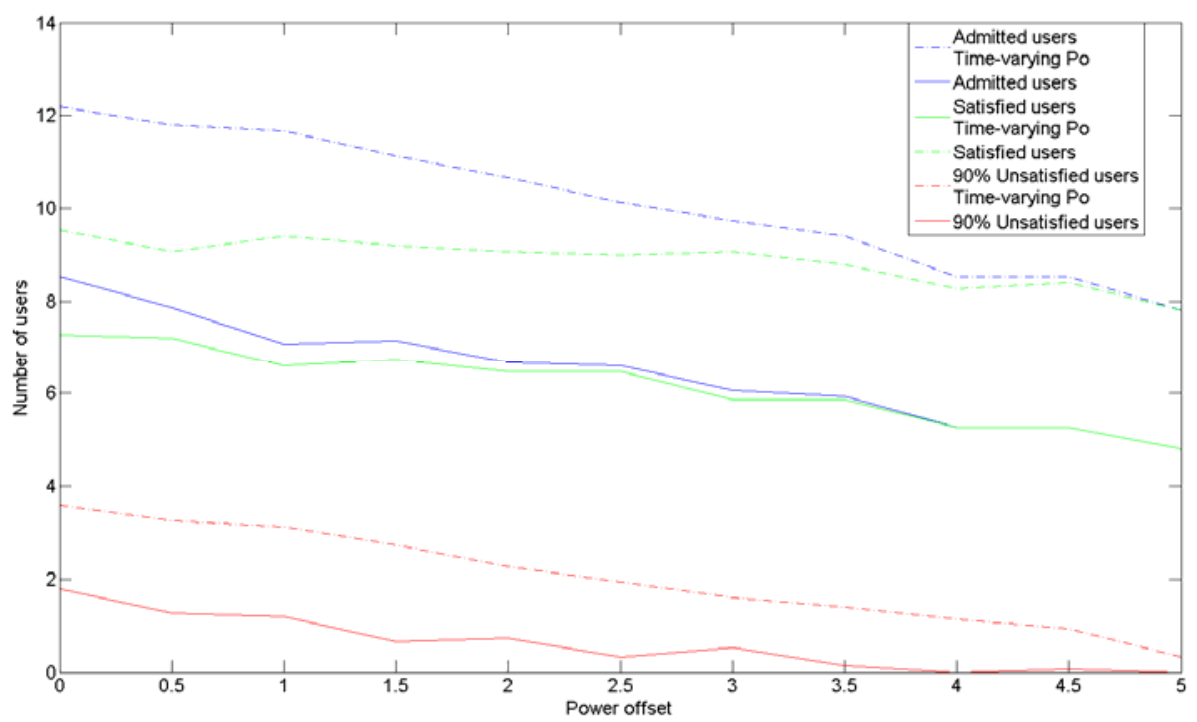

Figure 2. AC with and without time varying Po based algorithm 
Depending on the policies deployed by the operator, if we give the same importance to the blocked users than the non satisfied users, admission control algorithms based on giving priority to the users closest to the Node-B improves users QoS, decreasing the number of blocked users, which results in higher incomings for the operator company (see Figure 4). However, this CAC can be seen as unfair, since those users far from the node B will be accepted less frequently than those nearer, thus affecting radio planning methods.

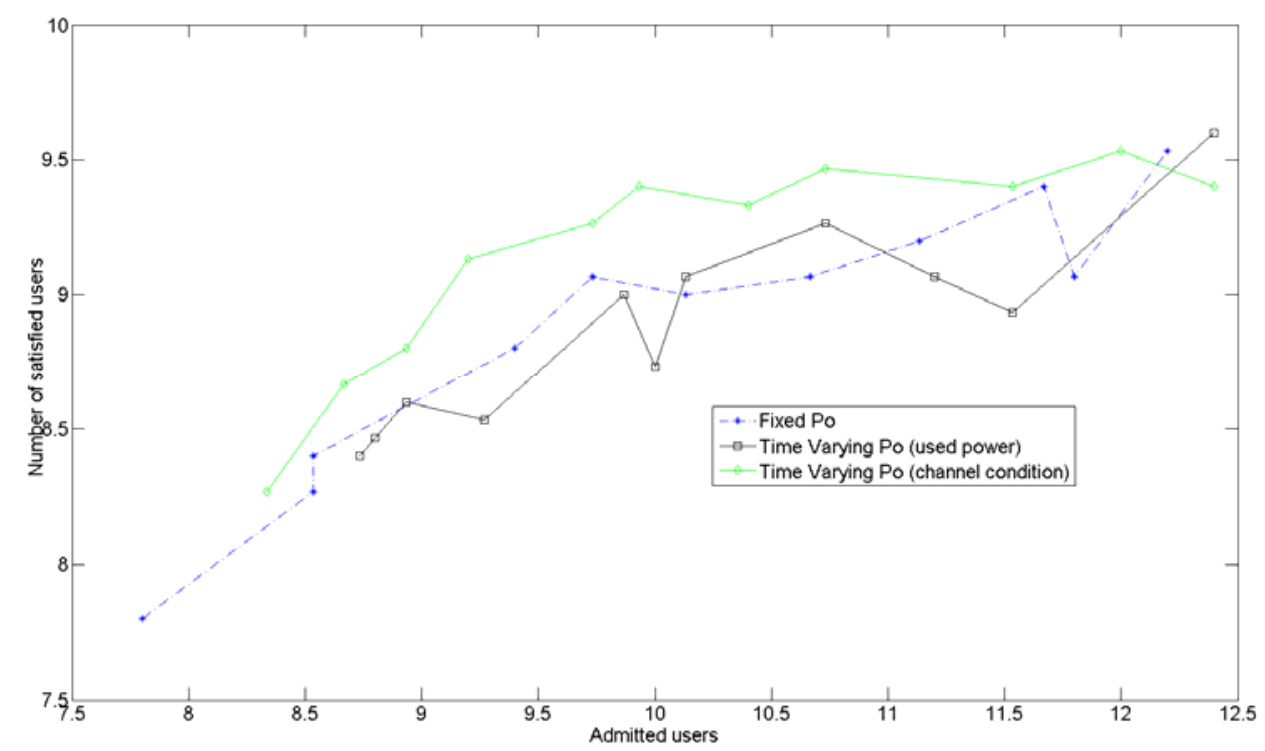

Figure 3. Satisfied users vs admitted users

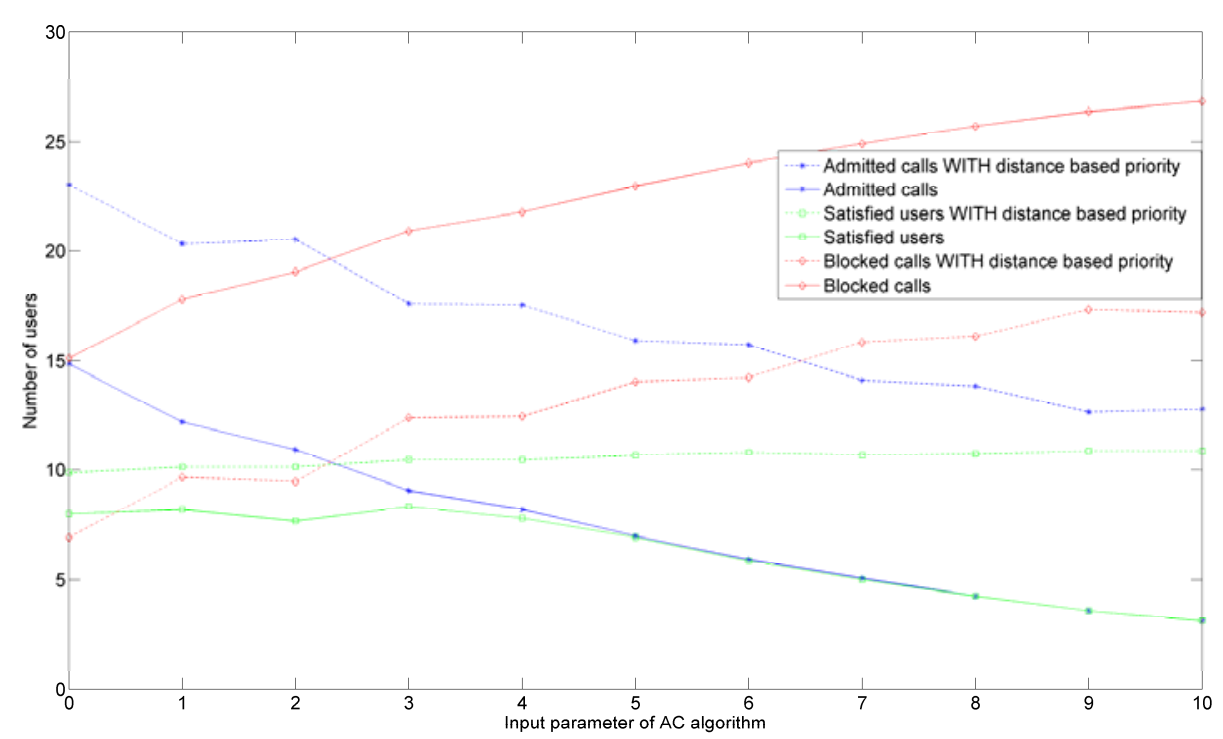

Figure 4. Time-varying power reservation based on channel conditions

\section{Conclusions}

We have analyzed the performance of three QoS oriented call admission control 
algorithms, which differ on the way they reserve power for the admitted calls. We evaluated the performance of these algorithms together with a strict channel allocation policies scheduling algorithm. We proposed two new ways to make the power reservation variable in time. We compared them with the one in [3] using a HSDPA CAC simulator developed in Matlab. Our results show that with a simple implementation of these algorithms the system doesn't achieve high improvement. However, if we give higher priority to the calls with better channel conditions (near to the cell), the satisfied user probability is higher with the implementations of our algorithm. Our results show that giving priority to users nearest the Node-B gives us a higher number of admitted calls and satisfied calls. The best results obtained from our simulations was the one that combines the algorithm that we propose, that make time-varying power reservation based on channel quality, with giving priority to the nearest users.

\section{Acknowledgement}

The research has been partially done under the scope of the IPARHAIZE research project (Regional research project funded the Basque Government (Spain)).

\section{References}

[1] ETSI, T. (2002). TS 25.301, Universal Mobile Telecommunications Systems (UMTS): Radio Interface Protocol Architecture (3GPP TS 25.301 version 5.1. 0 Release 5).

[2] Holma, H., \& Toskala, A. (2006). HSDPA/HSUPA for UMTS: High speed radio access for mobile communications: John Wiley \& Sons, Maiden, MA, US.

[3] Pedersen, K. (2005). Quality based HSDPA access algorithms. Paper presented at the IEEE 62nd Vehicular Technology Conference, VTC-2005-Fall, September 2005. Dallas, US.

[4] Holma, H., \& Toskala, A. (2000). WCDMA for UMTS: Radio access for third generation mobile communications: John Wiley \& Sons, Inc. New York, NY, USA.

[5] Niyato, D., \& Hossain, E. (2005). Call admission control for QoS provisioning in 4G wireless networks: issues and approaches. IEEE network, 19(5), 5-11.

[6] Elayoubi, S., Chahed, T., \& Hebuterne, G. (2007). Mobility-Aware Admission Control Schemes in the Downlink of Third-Generation Wireless Systems. IEEE Transactions on Vehicular Technology, 56(1), 245-259.

[7] Gomes, J., Choi, H., Kim, J., Sohn, J., \& Choi, H. (2007). Integrating admission control and packet scheduling for quality controlled streaming services in hsdpa networks. Paper presented at the Fourth International Conference on Broadband Communications, Networks and Systems, BROADNETS 2007, September 2007. Raleigh, North Carolina, US.

[8] Barriac, G., \& Holtzman, J. (2002). Introducing delay sensitivity into the proportional fair algorithm for CDMA downlink scheduling. Paper presented at the IEEE Seventh International Symposium on Spread Spectrum Techniques and Applications, September 2002. Prague, Czech Republic. 